\title{
COMPOSIÇÃO QUÍMICA E CONTEÚDO DE FERRO SOLÚVEL EM SOJA [Glycine max (L.) Merrill]
}

\author{
LETÍCIA TAMIE PAIVA YAMADA ${ }^{1}$ \\ MARIA DE FÁTIMA PÍCCOLO BARCELOS ${ }^{2}$ \\ RAIMUNDO VICENTE DE SOUSA ${ }^{2}$ \\ ANDRELISA LINA DE LIMA ${ }^{3}$
}

\begin{abstract}
RESUMO - A soja é um grão que possui um dos maiores teores de proteína e ferro entre os alimentos de origem vegetal e teve a sua utilização muito aumentada nos últimos anos, principalmente por lactentes que têm problemas de intolerância ao leite de vaca em todo mundo. Em razão da sua grande quantidade de ferro, a soja tem sido investigada quanto à sua capacidade de recuperação de indivíduos anêmicos. No passado, a investigação baseou-se somente na sua quantidade de ferro total, não considerando as frações solúveis (mais biodisponíveis) das insolúveis (que são mais dificilmente absorvidas). Objetivou-se com este trabalho realizar análises químicas em quatro cultivares de soja Glycine $\max ($ L.) Merrill (IAC PL-1; IAC 22; IAC 8-2 E IAC 15-2), bem como os seus respectivos conteúdos de ferro solúvel. Os teores de proteína nos grãos, que foram em média de 34,46\%, foram estatisticamente iguais; o de lipídeos foi maior para a cultivar IAC PL-1, com
\end{abstract}

TERMOS PARA INDEXAÇÃO: Soja, composição centesimal, composição mineral e ferro solúvel.

\section{CHEMICAL COMPOSITION AND SOLUBLE IRON CONTENT IN SOYBEAN [Glycine max (L.) Merrill]}

\begin{abstract}
Soybean is a grain which possesses one of the highest contents of protein and iron among the foods of plant origin and had its use greatly increased in the latest years, in the eastern countries mainly by lactents who have problems of intolerance to bovine's milk. Due to its great concentration of iron, soybean has been investigated as to its capacity of recovering anemic individuals. In the past, the investigation was based only on its concentration of total iron, not taking into account the soluble fractions (greater bioavailability) from the insoluble (which are less bioavailable). This work was designed to accomplish chemical analyses on four soybean cultivars Glycine max (L.) Merrill (IAC
\end{abstract}

$20,07 \%(\mathrm{P}<0,01)$ e a cultivar que apresentou o menor teor de fibras foi também a IAC PL-1, com 5,24\% $(\mathrm{P}<0,01)$, no grão moído e tratado termicamente a $95^{\circ} \mathrm{C}$ por uma hora. A quantidade de ferro total foi maior para a cultivar IAC 8-2, com 110,27 ppm, seguida pela IAC PL-1, que apresentou uma média de 101,93 ppm de ferro. Quanto aos seus conteúdos de ferro solúvel e de polifenóis, não houve diferença estatística entre os tratamentos, sendo os seus conteúdos médios de ferro solúvel cerca de 2,52 mg/100 g, e de tanino cerca de 5,51 $\mathrm{mg} / 100$ de soja moída e tratada termicamente. Pelos resultados dessas análises, verifica-se que a soja IAC PL1 seria a mais indicada para um possível tratamento de anemia ferropriva de ratos, por ter bons conteúdos de ferro total e conteúdo de ferro solúvel estatísticamente igual à da soja IAC 8-2, que teve o maior conteúdo de ferro total e por apresentar os menores teores de fibras.

\footnotetext{
1. Professora do Unicentro Newton Paiva.

2. Professores da UNIVERSIDADE FEDERAL DE LAVRAS/UFLA - Caixa Postal 37 - 37200-000 - Lavras, MG.

3. Acadêmica do Curso de Agronomia/ UFLA.
}

PL-1; IAC 22; IAC 8-2 and IAC 15-2), as well as their respective contents of soluble iron. Protein contents in the grains, that were on average $34,46 \%$ were statistically similar; oil content was greater for the cultivar IAC PL-1, with $20,07 \%(\mathrm{P}<0,01)$, the same cultivar which presented the lower fiber content, with $5,24 \%(\mathrm{P}<0,01)$ in the ground grain and heat -treated at $95^{\circ} \mathrm{C}$ for one hour. The amount of total iron was higher for the cultivar IAC 8-2 with $110,27 \mathrm{ppm}$, followed by IAC PL-1 which presented the mean of $101,93 \mathrm{ppm}$ of iron. Concerning their contents of soluble iron and polyphenols, there were no statistic differences among the 
treatments $(\mathrm{P}<0,01)$, their average contents of soluble iron of about $2,52 \mathrm{mg} / 100 \mathrm{~g}$ and of tannin about 5,51 $\mathrm{mg} / 100$ of ground and heat -treated soybean. The results of those analyses indicate that the soybean IAC PL-1 would be the most adequate for a possible treatment for ferropriva anemia of rats for having good contents of total iron and content of soluble iron statistically equal to that of the soybean IAC 8-2, which presented the greatest content of total iron and for presenting the poorest contents of fiber.

INDEX TERMS: Soybean, centesimal composition, mineral composition and soluble iron.

\section{INTRODUÇÃO}

A soja, no mundo oriental, é a base de composição de diversos pratos naturais e tradicionais, sendo, em certos casos, como de populações de baixa renda e vegetarianos, a principal fonte de proteína. Nos países ocidentais, é largamente utilizada na indústria alimentícia, sendo a sua aceitação como alimento natural ainda pequena, devido ao odor e sabor desagradáveis (Sediyama et al., 1989; Shaw et al., 1995; Morais \& Silva, 1996).

Muitos pesquisadores têm se empenhado no estudo dessa leguminosa, a qual possui importantes qualidades para a nutrição, como a grande quantidade de proteínas vegetais de alto valor biológico, além de ser fonte de ácidos graxos poliinsaturados (linoléico e linolênico). A soja ainda é rica em minerais, em especial magnésio, fósforo, cobre, zinco e ferro. A utilização desses minerais pode ser prejudicada por causa de fatores presentes no grão, os quais são considerados por muitos pesquisadores como antinutricionais (Gillooly et al., 1984; Latunde-Dada \& Neale, 1986), mas esses dados são ainda questionáveis, pois muitos resultados são conflitantes. Quanto ao ferro, diversos são os resultados encontrados.

O ferro dos alimentos de origem animal é mais bem absorvido do que o de alimentos de origem vegetal, mas são esses últimos que contribuem com $90 \%$ da ingestão dietética desse mineral nos países desenvolvidos e com até $100 \%$ nos países em desenvolvimento. Os alimentos de origem animal são caros e não estão acessíveis à maioria da população, representando apenas de $5 \%$ a $10 \%$ do ferro total de uma refeição, principalmente em populações pobres (Bianchi et al., 1992).

A soja apresenta, entre os produtos de origem vegetal, uma elevada quantidade de ferro (de 9 a 13 $\mathrm{mg} / 100 \mathrm{~g}$ ), que possui boa biodisponibilidade. O termo biodisponibilidade, relacionado ao ferro, é a medida daquela fração do ferro alimentar capaz de ser absorvida pelo trato gastrointestinal e subseqüentemente armazenada e incorporada ao grupo heme da hemoglobina (Bianchi et al., 1992).

Sabe-se que, apesar de muitos alimentos serem aparentemente boas fontes de ferro, não o são realmente pela sua disponibilidade biológica, que se dá em função de sua forma química e da presença de itens alimentares que promovam ou inibam sua absorção. Para que a absorção seja ótima, é necessário que os minerais e elementos traço estejam presentes na parte intraluminal na forma solúvel. A maioria dos fatores dietéticos que apresentam influência na disponibilidade desses nutrientes possui uma ação direta ou indireta sobre a solubilidade dos mesmos. Entre os inibidores da absorção do ferro, estão alguns dos componentes das fibras (hemicelulose e lignina), o ácido oxálico, o ácido fítico e outros polifenóis, e que estão presentes no grão da soja. Porém, os relatos sobre o efeito exato e o mecanismo de ação desses fatores são ainda muito controvertidos.

Considerando as inúmeras aplicações da soja, muitos esforços têm sido empregados para contornar o problema da biodisponibilidade de minerais. Um grupo de pesuisadores, Beard et al. (1996), analisou a eficácia da ferritina de algumas variedades de soja (uma forma solúvel de ferro presente no grão) na recuperação de ratos anêmicos e observaram que o conteúdo de ferritina variava de cultivar para cultivar, sugerindo que um melhoramento das cultivares que possuam uma maior concentração de ferritina nos grãos poderia ajudar no combate à anemia, visto que o ferro ligado à ferritina mostrou-se eficaz na recuperação de ratos anêmicos, pois parece se manter biodisponível para esses animais, alimentados com soja, escapando das possíveis complexações com os outros componentes dessa leguminosa.

Com base nesses dados, com o presente estudo visou-se a analisar quatro cultivares de soja desenvolvidas e melhoradas no Brasil, provenientes do Instituto Agronômico de Campinas (IAC), com objetivo de selecionar a cultivar que apresentasse os melhores níveis de ferro solúvel e de ferro total nos grãos.

\section{MATERIAL E MÉTODOS}

As análises foram realizadas nos laboratórios dos Departamentos de Ciência dos Alimentos, de Química e de Fitotecnia da Universidade Federal de Lavras (UFLA), Lavras, MG.

Foram utilizadas quatro cultivares de soja [Glicyne $\max (\mathrm{L}$.$) Merrill]: IAC PL-1, IAC 22, IAC 8-2$ 
e IAC 15-2, oriundas do Instituto Agronômico de Campinas (IAC), São Paulo, safra 1999 e que constituíram os quatro tratamentos, respectivamente. Foram realizadas análises químicas e bioquímicas de cada cultivar, todas em quadruplicata e o delineamento utilizado foi o inteiramente casualizado (DIC). Os dados foram submetidos à análise de variância e teste de $\mathrm{F}$, sendo os resultados significativos estudados por meio da comparação de médias pelo teste de Tukey, utilizando o programa estatísico MSTATC para as análises.

Os grãos de soja de cada cultivar foram selecionados e triturados integralmente (com o tegumento externo) até a obtenção de uma farinha fina. Logo depois, foram submetidos a um tratamento térmico de $95^{\circ} \mathrm{C}$ por uma hora, em estufa a vácuo, para diminuir em níveis desejáveis a ação dos inibidores de tripsina e das lectinas. Após esse tratamento, a farinha foi armazenada a $15^{\circ} \mathrm{C}$, para análises posteriores.

Toda a vidraria e demais utensílios utilizados nas análises foram lavados com ácido clorídrico puro e enxagüados utilizando-se água destilada, deionizada e desmineralizada (ultrapura). Toda a água utilizada nas análises foi ultrapura, para evitar contaminação de ferro provenientes de outras fontes.

$\mathrm{O}$ teor de umidade foi determinado por dessecação em estufa a $105^{\circ} \mathrm{C}$, segundo AOAC (1990). O teor de óleo foi determinado utilizando-se extrator contínuo tipo Soxhlet, segundo a AOAC (1990). O restante das análises foi determinado nas amostras secas e desengorduradas: a proteína bruta foi determinada segundo método de Kjeldhal (AOAC, 1990) e multiplicado pelo fator de 6,25. A quantidade de fibra bruta foi determinada pelo método de Van de Kamer \& Van Ginkel (1952). Finalmente, o teor de extrato não nitrogenado (ENN) foi obtido por diferença e o de cinzas (resíduo mineral fixo), determinado por incineração do material em forno tipo mufla a $500^{\circ} \mathrm{C}$, segundo a AOAC (1990).

Os minerais foram determinados submetendo primeiramente as amostras a uma digestão com solução nitro-perclórica (2:1) e quantificados por espectrofotometria de absorção atômica, com as leituras feitas em aparelho da marca Varian, Spectr AA, 100; conforme a AOAC (1990), usando lâmpada específica.

A concentração de ferro solúvel foi determinada utilizando-se um método in vitro, adaptado de Politz \& Clydesdale (1988) e Slatkavitz \& Clydesdale (1988), citados por Nkunzimana et al. (1996). O método baseia-se em submeter uma quantidade conhecida de amostra a uma digestão péptica, seguida de digestão pancreática, em condições controladas de tempo, temperatura e $\mathrm{pH}$.
A pepsina foi suspensa em $25 \mathrm{~mL}$ de $\mathrm{HCl} 0,1 \mathrm{~N}$. A pancreatina purificada e o extrato de bile foram dissolvidos em $250 \mathrm{~mL}$ de solução tampão de Tyrode, que continha os seguintes reagentes: $2 \mathrm{~g}$ de $\mathrm{NaCl} ; 0,05 \mathrm{~g}$ de $\mathrm{KCl} ; 0,065 \mathrm{~g}$ de $\mathrm{MgSO}_{4} .7 \mathrm{H}_{2} \mathrm{O} ; 0,25 \mathrm{~g}$ de glucose; $0,25 \mathrm{~g}$ de $\mathrm{NaHCO}_{3} ; 0,066 \mathrm{~g}$ de $\mathrm{CaCl}_{2} ; 0,05 \mathrm{~g}$ de $\mathrm{NaN}_{3}$. Esse tampão foi ajustado para $\mathrm{pH} 5$, com $\mathrm{HCl} 6 \mathrm{~N}$.

As respectivas quantidades de pepsina, pancreatina e bile foram determinadas de acordo com o conteúdo de proteína das farinhas, previamente determinadas pelo método Kjeldhal (Nx 6,25). A razão pepsina para proteína foi de 1:50, a de pancreatina para proteína, de 1:30 e a de bile para proteína, 1: 19,2. A digestão realizou-se em incubadora a $37^{\circ} \mathrm{C}$, por $2 \mathrm{~h}$, em $\mathrm{pH}=2$, para simular as condições gástricas. A cada $35 \mathrm{~min}, \mathrm{o} \mathrm{pH}$ foi ajustado com $\mathrm{NaOH} 1 \mathrm{~N}$. Após a digestão péptica, o $\mathrm{pH}$ foi aumentado para 5 , com $\mathrm{NaOH} 3 \mathrm{~N}$ e a mistura de pancreatina e extrato de bile foi adicionada. A incubação continuou por mais duas horas, e ao final dessas, o digerido foi centrifugado em tubos de $250 \mathrm{~mL}$ a 10.000 $\mathrm{g}$ por $20 \mathrm{~min}$, a $4^{0} \mathrm{C}$ e o sobrenadante filtrado em papel de filtro whatman \# 41. O conteúdo de ferro solúvel foi então determinado nesse sobrenadante por espectrofotometria de absorção atômica e as concentrações de ferro foram lidas diretamente a $248 \mathrm{~nm}$, segundo a AOAC (1990).

Os taninos (polifenóis) foram analisados utilizando-se o método colorimétrico de Folin-Denis, conforme a AOAC (1990) e Deshpande et al. (1986). A extração foi feita com metanol a $80 \%$ em banho-maria $\left(80^{\circ} \mathrm{C} / 15\right.$ minutos $)$, utilizando-se pérolas de vidro e varetas de refluxo, por três vezes. Os extratos foram, então, reunidos e evaporados até cerca de $25 \mathrm{~mL}$ e submetidos à dosagem de polifenóis, empregando o ácido tânico como padrão.

\section{RESULTADOS E DISCUSSÃO}

\section{Composição centesimal}

A composição química das cultivares analisadas (Tabela 1) encontra-se de acordo com os padrões observados na literatura (Smith \& Circle, 1978; Morais \& Silva, 1996). Com relação à soja cultivada no Brasil, Castro et al. (1973) verificaram que a quantidade de proteína varia de $29,2 \%$ a $57,9 \%$ e a de lipídeos varia de $14,7 \%$ a $28,4 \%$, de acordo com a variedade. As cultivares apresentaram, em média, 34,46\% de proteína, não diferindo estatisticamente entre si $(\mathrm{P}<0,01)$. A IAC PL1 foi a que apresentou o maior teor de óleo em relação às outras cultivares. 
Quanto às fibras, a cultivar IAC PL-1 foi a que apresentou as menores quantidades, e a IAC PL 8-2, a que apresentou as maiores quantidades. $\mathrm{O}$ termo fibra dietética define um conjunto de diferentes compostos, entre eles a hemicelulose e a lignina, consideradas como complexantes de ferro e outros minerais em um experimento realizado por Gillooly et al. (1984). De acordo com Dintzis et al. (1979), o tegumento externo da soja contém $87 \%$ das fibras desse grão, dos quais $40 \%$ a $53 \%$ são de celulose, $14 \%$ a $33 \%$ de hemicelulose e 1 a $3 \%$ de lignina. De acordo com esses autores, o simples fato de não se utilizar esses tegumentos externos da soja poderia diminuir em muito o teor de fibras e, consequientemente, aumentar a proporção de ferro e de outros minerais que seriam absorvidos.

\section{Composição mineral}

As concentrações de minerais das cultivares encontram-se expressas nas Tabelas 2 e 3. Pode-se notar que houve diferença significativa no teor de ferro total entre as amostras de soja, tendo a cultivar IAC 8-2 a maior média desse mineral $(110,27 \mathrm{ppm})$, seguida pela cultivar IAC PL-1 (101,93ppm). As cultivares IAC 22 e IAC 15-2 apresentaram os menores índices de ferro total nos grãos. Quanto ao zinco e fósforo, a IAC PL-1 apresentou os maiores teores, e a IAC 15-2, os menores. Para o cálcio e o magnésio, as cultivares IAC PL-1, IAC 22 e a IAC 8-2 tiveram os maiores valores, e a IAC 8-2 não diferiu da IAC 15-2, que apresentou as menores quantidades. Para o manganês e o enxofre, os maiores teores foram os das cultivares IAC 22 e IAC 8-2. Para o potássio, somente a IAC 8-2 foi diferente, apresentando as menores quantidades. Em relação ao cobre, não houve diferença estatística entre as cultivares $(\mathrm{P}<0,05)$.

TABELA 1 - Composição centesimal de quatro cultivares de soja.

\begin{tabular}{lcccccc}
\hline Tratamentos & Umidade $(\boldsymbol{\%})$ & Óleo $^{\mathbf{1}}(\boldsymbol{\%})$ & Proteína $^{\mathbf{1}}(\boldsymbol{\%})$ & Fibras $^{\mathbf{1}}(\boldsymbol{\%})$ & Cinza $^{\mathbf{1}}(\boldsymbol{\%})$ & ENN $^{\mathbf{2}}(\boldsymbol{\%})$ \\
\hline IAC PL-1 & $8,35 \mathrm{a}$ & $20,07 \mathrm{a}$ & $34,97 \mathrm{a}$ & $5,24 \mathrm{~b}$ & $4,61 \mathrm{~b}$ & 25,86 \\
IAC 22 & $8,18 \mathrm{a}$ & $18,45 \mathrm{~b}$ & $33,42 \mathrm{a}$ & $5,61 \mathrm{ab}$ & $5.19 \mathrm{a}$ & 29,20 \\
IAC 8-2 & $7,50 \mathrm{~b}$ & $18.63 \mathrm{~b}$ & $35,07 \mathrm{a}$ & $6,58 \mathrm{a}$ & $4,14 \mathrm{c}$ & 27,14 \\
IAC 15-2 & $8,49 \mathrm{a}$ & $18,87 \mathrm{~b}$ & $34,38 \mathrm{a}$ & $5,98 \mathrm{ab}$ & $4,11 \mathrm{c}$ & 27,38 \\
\hline Teste F & $11,533 * *$ & $6,834 * *$ & $1,015 \mathrm{~ns}$ & $5,739 * *$ & $24,23 * *$ & - \\
C.V. & 3,19 & 2,95 & 4,37 & 8,13 & 4,57 & - \\
\hline
\end{tabular}

${ }^{1}$ dados expressos com base em matéria seca.

${ }^{2}$ ENN = extrato não nitrogenado (obtido por diferença).

**F significativo a $1 \%$.

*F significativo a $5 \%$.

Médias seguidas pelas mesmas letras não diferem entre si, a $5 \%$ de probabilidade, pelo teste Tukey.

TABELA 2 - Teores médios de cobre, manganês, zinco e ferro de quatro cultivares de soja.

\begin{tabular}{lcccc}
\hline \multicolumn{1}{c}{ Tratamentos } & Cu mg/kg & Mn mg/kg & Zn mg/kg & Fe mg/kg \\
\hline IAC PL1 & $10,46 \mathrm{a}$ & $20,27 \mathrm{bc}$ & $42,33 \mathrm{a}$ & $101,93 \mathrm{~b}$ \\
IAC 22 & $7,10 \mathrm{a}$ & $24,17 \mathrm{ab}$ & $40,17 \mathrm{~b}$ & $88,00 \mathrm{c}$ \\
IAC 8-2 & $10,50 \mathrm{a}$ & $24,83 \mathrm{a}$ & $40,00 \mathrm{~b}$ & $110,27 \mathrm{a}$ \\
IAC 15-2 & $6,97 \mathrm{a}$ & $18,43 \mathrm{c}$ & $37,80 \mathrm{c}$ & $84,50 \mathrm{c}$ \\
\hline Teste F & 6,049 & $11,539 * *$ & $51,664 * *$ & $45,291 * *$ \\
C.V. & 16,02 & 7,16 & 1,11 & 3,22 \\
\hline
\end{tabular}

**F significativo a $1 \%$

*F significativo a $5 \%$

Médias seguidas pelas mesmas letras não diferem entre si, a 5\% de probabilidade, pelo teste Tukey. 
TABELA 3 - Teores médios de fósforo, potássio, cálcio, magnésio e enxofre de quatro cultivares de soja.

\begin{tabular}{lccccc}
\hline \multicolumn{1}{c}{ Tratamento } & $\boldsymbol{\%} \mathbf{P}$ & $\mathbf{\%} \mathbf{~ K}$ & $\mathbf{\%} \mathbf{C a}$ & $\mathbf{\%} \mathbf{M g}$ & $\boldsymbol{\% ~} \mathbf{~}$ \\
\hline IAC PL1 & $0,58 \mathrm{a}$ & $2,06 \mathrm{a}$ & $0,19 \mathrm{a}$ & $0,27 \mathrm{a}$ & $0,37 \mathrm{~b}$ \\
IAC 22 & $0,48 \mathrm{c}$ & $2,07 \mathrm{a}$ & $0,20 \mathrm{a}$ & $0,26 \mathrm{a}$ & $0,41 \mathrm{a}$ \\
IAC $8-2$ & $0,51 \mathrm{~b}$ & $1,94 \mathrm{~b}$ & $0,18 \mathrm{ab}$ & $0,27 \mathrm{a}$ & $0,39 \mathrm{ab}$ \\
IAC 15-2 & $0,42 \mathrm{~d}$ & $2,01 \mathrm{ab}$ & $0,17 \mathrm{~b}$ & $0,24 \mathrm{~b}$ & $0,37 \mathrm{~b}$ \\
\hline Teste F & $280,445^{* *}$ & $11,872^{* *}$ & $7,394^{*}$ & $19,889^{* *}$ & $10,788^{* *}$ \\
C.V. & 1,42 & 1,40 & 5,08 & 1,93 & 2,49 \\
\hline
\end{tabular}

**F significativo a $1 \%$

*F significativo a $5 \%$

Médias seguidas pelas mesmas letras não diferem entre si, a 5\% de probabilidade, pelo teste Tukey

A soja é uma fonte moderada de cálcio. Muitos autores que compararam o "leite" de soja (extrato solúvel de soja) com o leite de vaca observaram valores de biodisponibilidade de cálcio que chegavam a ser de $90 \%$ dos valores do leite de vaca. Isso não ocorreu com a soja integral assada, na qual somente $10 \%$ do cálcio puderam ser efetivamente utilizados (Schroeder et al. e Desikachar et al., citados por Smith \& Circle, 1978). Comparando-se os valores de cálcio encontrados com o de outras fontes alimentares, como o arroz $(10 \mathrm{mg} / 100 \mathrm{~g})$, o feijão vermelho cozido (38 mg/100g), a farinha de mandioca $(148 \mathrm{mg} / 100 \mathrm{~g})$ e o milho $(6 \mathrm{mg} / 100 \mathrm{~g})$, observa-se que a soja, que teve em média $185 \mathrm{mg} / 100 \mathrm{~g}$, é o alimento que possui valores maiores desse mineral (Azoubel et al., 1982).

A soja é um alimento de origem vegetal que possui grandes quantidades de ferro. Fonseca (1995) e Bianchi et al. (1992) citaram em seus trabalhos que o ferro da soja foi mais absorvido que o do trigo, milho, feijão preto e arroz (em ordem decrescente). Comparando-se os valores aqui encontrados, de 8,45 a $11,03 \mathrm{mg} / 100 \mathrm{~g}$, com os do feijão preto, $4,3 \mathrm{mg} / 100$ $\mathrm{g}$, feijão vermelho, 7,10 mg/100 g, fubá, 1,8 mg/100 $\mathrm{g}$, farinha de mandioca, $5,4 \mathrm{mg} / 100 \mathrm{~g}$ e trigo, 0,9 $\mathrm{mg} / 100 \mathrm{~g}$, verifica-se que todos estes produtos, utilizados com frequiência na alimentação humana, apresentam teores de ferro inferiores ao da soja (Azoubel et al., 1982).

Comparando-se os valores de ferro total das cultivares IAC 8-2 (110,27 ppm) e IAC PL-1 (101,93 ppm), com as cultivares Tokyo (80,98 ppm) e Jackson (81,54 ppm), analisados por Beard et al. (1996), percebe-se que foram superiores nas cultivares do IAC.

\section{Ferro solúvel}

Com relação à concentração de ferro solúvel, não houve diferença significativa entre os tratamentos (Figura 1).

Técnicas mais apuradas para detectar a concentração de ferro solúvel poderiam revelar diferenças entre essas cultivares, como foi observado por Beard et al. (1996).

Comparando-se esses valores de ferro solúvel com os valores de ferro total, verifica-se que realmente há uma grande diferença entre os tipos de ferro presentes no grão de soja. E, aparentemente, somente $26,7 \%$ do ferro total presente nos grãos das cultivares analisadas estão disponíveis para a absorção, ou seja, permanecem solúveis na luz intestinal.

A média dos teores de ferro solúvel das cultivares analisadas $(2,52 \mathrm{mg} / 100 \mathrm{~g})$ é suficiente para suprir as necessidades de ferro de mulheres adolescentes $(2,02 \mathrm{mg} / \mathrm{dia})$; homens e mulheres adultos $(1,14$ $\mathrm{mg} /$ dia e $2,38 \mathrm{mg} /$ dia respectivamente), com exceção das gestantes, e atendem a $16,8 \%$ da quota dietética recomendada (RDA) para mulheres de 14 a 18 anos, e a $14 \%$ da RDA para mulheres de 19 a 50 anos (Dietary Reference Intakes, 2001). O termo "necessidade" é definido como a quantidade que se deve absorver para repor as perdas do organismo e

que seja suficiente para cobrir o aumento normal da necessidade durante o crescimento e gestação, ao passo que o termo quota dietética recomendada (recommended dietary allowances) refere-se à meta de ingestão de nutrientes, provenientes da dieta, para indivíduos saudáveis (OMS/FAO, 1991). 
Beard et al. (1996) também observaram essas diferenças quando analisaram variedades de soja cultivadas nos EUA. Em seus resultados, cerca de $45,28 \%$ do ferro total estavam na fração solúvel. Essa maior porcentagem, no entanto, não significa necessariamente que as cultivares do IAC tenham menores quantidades de ferro solúvel que as dos EUA, pois as técnicas utilizadas para a determinação desse tipo de ferro foram diferentes. Segundo Ragland \& Theil (1993), a fração solúvel de ferro das sementes da soja contém dois tipos de ferro: a ferritina e o fitato monoférrico. Segundo Beard et al. (1996), a ferritina correspondeu a $30 \%$ do total do ferro solúvel. Esses pesquisadores também observaram que tal quantidade de ferro pode ser modificada por melhoramento genético, para se obterem cultivares com maiores quantidades de ferritina, o que poderia melhorar a biodisponibilidade do ferro na soja.

\section{Polifenóis (taninos)}

As concentrações médias dos taninos encontradas nas quatro variedades de soja apresentam-se na Figura 2.
Não houve diferença estatística significativa entre os tratamentos, e a média de taninos presente nas cultivares foi de $5,51 \mathrm{mg} / 100 \mathrm{~g}$.

Os taninos são considerados potentes inibidores da absorção de ferro e de outros minerais, como o zinco e o cálcio (Latunde-Dada \& Neale, 1986). Segundo Liener (1994), apesar de presentes na soja, a quantidade de taninos nesse grão não é considerada suficiente para provocar um efeito antinutricional. Hurrel et al. (1999) observaram uma redução de $50 \%$ a $70 \%$ na absorção de ferro em humanos, quando esses consumiram bebidas contendo cerca de 20 a $50 \mathrm{mg}$ de polifenóis totais em $275 \mathrm{ml}$ da bebida. No chá preto, ou de ervas, a concentração de taninos média foi de $11,67 \mathrm{mg} / \mathrm{g}$ de chá, ao passo que a média desse polifenol na soja IAC foi de $0,055 \mathrm{mg} / \mathrm{g}$ de soja integral, ou seja, $0,47 \%$ do valor dos taninos presentes nos chás analisados naquele experimento. Essa quantidade de taninos encontrada nas cultivares do IAC não interfere de forma potente na absorção do ferro dessas cultivares de soja.

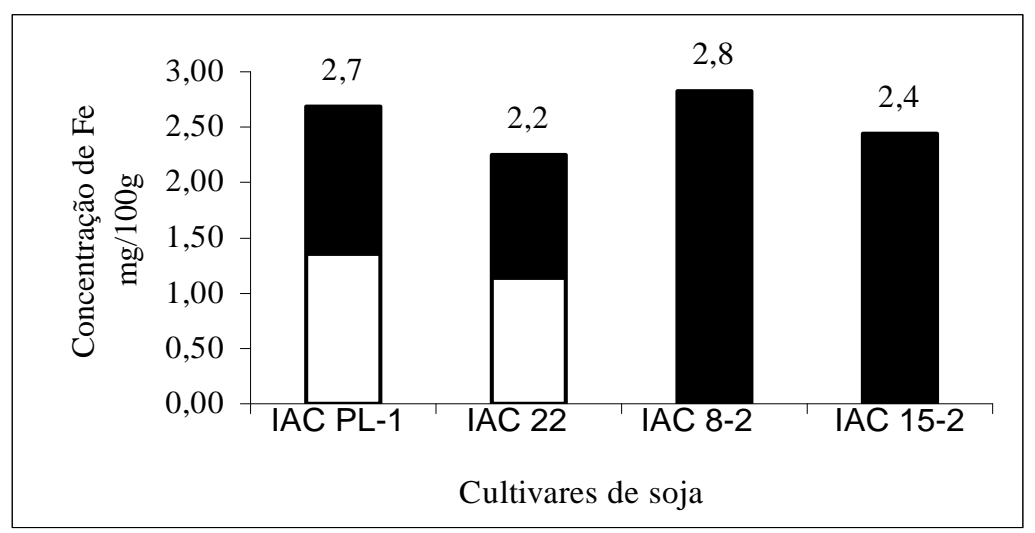

FIGURA 1 - Teores de ferro solúvel (mg/100g) de quatro cultivares de soja. 


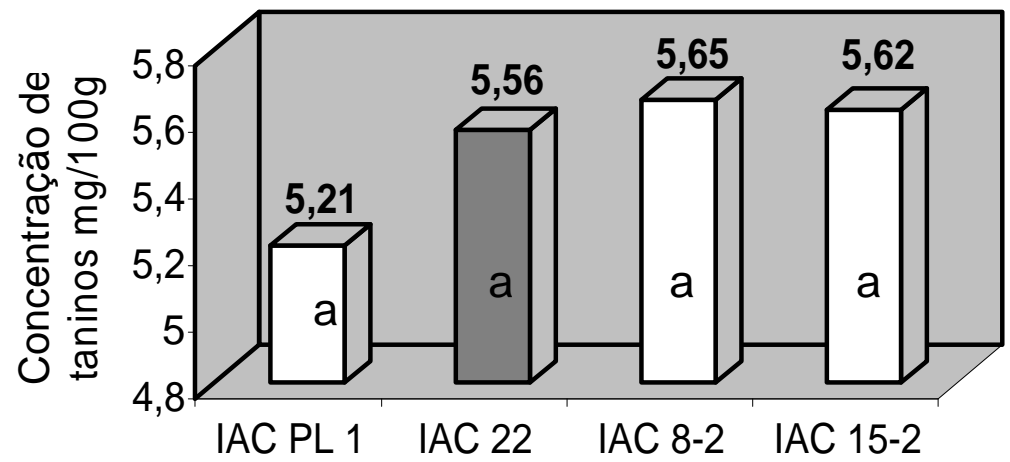

Cultivares de soja

FIGURA 2 - Teores de taninos $(\mathrm{mg} / 100 \mathrm{~g})$ de quatro cultivares de soja.

\section{CONCLUSÕES}

Das cultivares de soja analisadas neste trabalho, a IAC 8-2 e a IAC PL-1 parecem ser as mais indicadas para utilização na alimentação humana como fonte de ferro, mais especificamente para populações com anemia ferropriva. Isso porque, em termos de teores de ferro total, seus conteúdos foram os mais altos e, apesar de a cultivar IAC 8-2 apresentar valores mais altos que a IAC PL-1, os teores de ferro solúvel (mais biodisponível) foram estatisticamente iguais. Além disso, a cultivar IAC PL-1 apresentou ainda os menores teores de fibras. Com relação aos outros minerais, essas duas cultivares também apresentaram as maiores quantidades quando comparadas à IAC 22 e à IAC 15-2.

A quantidade de taninos (polifenóis) presentes nessas cultivares foi baixa e, provavelmente, insuficientes para provocar uma inibição significativa da absorção de ferro.

\section{REFERÊNCIAS BIBLIOGRÁFICAS}

ASSOCIATION OF OFFICIAL ANALYTICAL CHEMISTS. Official methods of analysis. 15. ed. Washington, 1990. v. 1-2.

AZOUBEL, L. N. O.; GARCIA, R. W. D.; NAVES, M. M. V. Tabela de composição de alimentos. In: OLIVEIRA, J. E. D. de; SANTOS, A. C.; WILSON, E. D. Nutrição básica. São Paulo: Sarvier, 1982. p. 249268.

BEARD, J. L.; BURTON, J. W.; THEIL, E. C. Purified ferritin and soybean meal can be sources of iron for treatingiron deficiency in rats. Journal of Nutrition, Philadelphia, v. 126, p. 154-160, 1996.

BIANCHI, M. L. P.; SILVA, H. C.; OLIVEIRA, J. E. D. Considerações sobre a biodisponibilidade do ferro do alimentos. Archivos Latino-americanos de Nutricion, Caracas, v. 42, n. 2, p. 94-100, 1992.

CAstro, A. T. B.; Millan, A.; LAGO, R. C. A. Contribuição ao estudo da soja no Brasil. Rio de Janeiro: Centro de Tecnologia Agrícola e Alimentar/Ministério da Agricultura, 1973. 28 p. (Boletim técnico, 10).

DESHPANDE, S. S.; CHERYAN, M.; SALUNKHE, D. K. Tannin analysis of food products. Critical Reviews in Food Science and Nutrition, Cleveland, v. 24, n. 4, p. 401-449, 1986.

DIETARY REFERENCE INTAKES. Applications in dietary Assessement. [S.1.: s.n.], 2001. 306 p.

DINTZIS, F. R.; LEGG, L. M.; DEATHERAGE, W. L.; BAKER, F. L.; INGLETT, G. E.; JAKOB, R. A.; RECK, S. J.; MUÑOZ, J. M.; KLEVAY, L. M.; SANDSTEAD, H. H.; SHUEY, W. C. Human gastrointestinal action on wheat, corn, and soy-hull bran: preliminary findings. Cereal Chemistry, Saint Paul, v. 56, p. $123-127,1979$.

FONSECA, J. G. M. Metabolismo de ferro e eritrócitos. In: VIEIRA, E. C.; FIGUEIREDO, E. A.; ALVAREZLEITE, J. I.; GOMEZ, M. V. Química fisiológica. 2. ed. São Paulo: Atheneu, 1995. p. 323-333. 
GILLOOLY, M.; BOTHWELL, T. H.; CHARLTON, R. W.; TORRANCE, J. D.; BEZWODA, W. R.; MACPHAIL, A. P.; DERMAN, D. P. Fators affeting the absorption of iron form cereals. British Journal of Nutrition, Cambridge, v. 51, p. 37-46, 1984.

HURRELL, R. F.; REDDY, M.; COOK, J. D. Inibition of non-haem iron absorption in mam by polyphenoliccontaining beverages. British Journal of Nutrition, Cambridge, v. 81, p. 289-295, 1999.

LATUNDE-DADA, G. O.; NEALE, R. J. Review: availability of iron from foods. Journal of Food Technology, Oxford, v. 21, p. 255-268, 1986.

LIENER, I. E. Implications of antinutritional componentes in soybean foods. Critical Reviews in Food Science and Nutrition, Cleveland, v. 1, n. 34, p. 31-67, 1994.

MORAIS, A. A. C.; SILVA, A. L. Soja e suas aplicações. Rio de Janeiro: Medsi, 1996. 259 p.

NKUNZIMANA, J.; ZEE, J. A.; TURGEONO'BRIEN, H.; MARIN, J. Potential iron bioavailability in usual diets of the imbo region of burundi. Journal of Agriculture and Food Chemistry, [S.1.], v. 44, p. 3591-3594, 1996.
ORGANIZAÇÃO MUNDIAL DA SAÚDE. ORGANIZAÇÃO DAS NAÇÕES UNIDAS PARA A AGRICULTURA E ALIMENTAÇÃO. Necesidades de vitamina $A$, hierro, folato y vitamina B12: informe de una consulta mixta FAO/OMS de expertos. Roma, 1991. $121 \mathrm{p}$.

RAGLAND, M.; THEIL, E. C. Ferritin and iron are developmentally regulates in nodules. Plant Molecular Biology, Dordrecht, v. 21, p. 555-560, 1993.

SEDIYAMA, T.; PEREIRA, M. G.; SEDIYAMA, C. S.; GOMES, J. L. L. Cultura da Soja. Viçosa: UFV, 1989. $75 \mathrm{p}$.

SHAW, N.; CHIN, C.; PAN, W. A vegetarian diet rich in soybean products compromises iron status in young students. Journal of Nutrition, Philadelphia, v. 125, p. 212-219, 1995.

SMITH, A. K.; CIRCLE, S. J. Soybeans: chemistry and technology. Connecticut: Avi Westport, 1978. v. 1, 470 p.

VAN DE KAMER, J. H.; VAN GINKEL, L. Rapid determination of crude fiber in cereals. Cereal Chemistry, Saint Paul, v. 29, n. 4, p. 239-251, July 1952. 